\title{
Kinetics of Chain Transfer Agents in Photopolymer Material
}

\author{
Jinxin Guo ${ }^{1}$, Michael R. Gleeson ${ }^{2}$, and John T. Sheridan ${ }^{1 *}$ \\ ${ }^{1}$ School of Electrical, Electronic and Communications Engineering, \\ College of Engineering and Architecture, \\ Communications and Optoelectronic Research Centre, \\ The SFI-Strategic Research Cluster in Solar Energy Conversion, \\ University College Dublin, Belfield, Dublin 4, Ireland. \\ ${ }^{2}$ Department of Computer Science, National University of Ireland Maynooth, \\ Maynooth, Co. Kildare, Ireland. \\ *Corresponding author: john.sheridan@ucd.ie; Tel: +353 (0)1 7161927
}

\begin{abstract}
The Non-local Photo-Polymerization Driven Diffusion (NPDD) model was introduced to describe the observed drop-off in the material's response for higher exposing spatial frequencies. Recent work carried out on the modeling of the mechanisms which occur in photopolymers during- and post-exposure, has led to the development of a tool, which can be used to predict the behaviour of these materials under a wide range of conditions. In this article, based on the chemical reactions of chain transfer agents, we explore this extended NPDD model, illustrating some of the useful trends, which the model predicts and we analyse their implications on the improvement of photopolymer material performance.
\end{abstract}

Keywords: Holography, photopolymer material, chain transfer agent.

\section{INTRODUCTION}

Photopolymer materials are being actively studied for practical applications such as holographic data storage, hybrid optoelectronics, photo embossing, including the manufacture of refractive and diffractive optical elements, and the selftrapping of light. ${ }^{1-4}$ Generally, in photopolymers, the photosensitizer absorbs light of an appropriate wavelength, becoming excited and causing the production of primary radicals, $R^{*}$. In free radical polymerization systems, the generation of $R^{*}$ leads to monomer being polymerized. During holographic exposure, a grating is formed by the interference fringe pattern. The resulting polymer chains formed grow away from their point of initiation, which results in a loss of recording fidelity and a reduction in the refractive index modulation amplitude. ${ }^{5}$ The Non-local PhotoPolymerization Driven Diffusion (NPDD) model was introduced to describe the observed decrease in the material's response for higher exposing spatial frequencies. ${ }^{6,7}$ The photopolymer's response at high spatial frequencies is very important as it determines the material's recording resolution and thus data storage capabilities. The NPDD model predicts that a reduction in the extent of the non-local effects within a material will result in an improvement in the high spatial frequency response and this prediction has been previously studied and experimentally confirmed. ${ }^{7,8}$

In order to maximise the potential of these materials, such as the addition of chain transfer agents (CTA), for various applications, the necessity of a physically comprehensive theoretical model of the effects which occur during photopolymerization is becoming ever more important. An extended NPDD model, which includes the effects of chain transfer and re-initiation kinetics, is presented in this paper. The provision of such a model will enable potential trends in a material's performance to be recognized and utilized. ${ }^{9,10}$ This will allow simulations of various ratios of key material components to be made, yielding indications of the most suitable material compositions required to increase material performance. 


\section{PHOTOCHEMICAL KINETIC MODEL}

\subsection{Photochemical process}

In the case of free radical photo-polymerization systems, the kinetic model describing what takes place involves five main processes, I. initiation, II. propagation, III. termination, IV. Inhibition, and V. chain transfer, each of which may involve several the physicochemical reactions. We highlight the major chemical reactions in each process below. ${ }^{11-15}$

\section{Initiation}

During illumination, the reaction between the photosensitiser and the electron donor (co-initiator) leads to the production of initiator radicals, $R^{\bullet}$, which can react with the monomers to produce chain initiators, $M^{\bullet}{ }^{13}$

$$
\begin{gathered}
I \stackrel{h v}{\longrightarrow} R^{\bullet}, \\
R^{\bullet}+M \stackrel{k_{i}}{\longrightarrow} M^{\bullet},
\end{gathered}
$$

where $I$ is the initiator, $h v$ indicates the energy absorbed from a photon, $k_{\mathrm{i}}$ is the chain initiation kinetic constant and $M$ represents a monomer molecule. The absorption process, which occurs during the initiation step, will be discussed in more detail in Section 2.2 .

\section{Propagation}

The chain initiator, $M_{1}^{\bullet}$, will attach itself to another monomer molecule, $M$, by addition to the $C=C$ bond yielding a growing polymer radical with an active tip. Through propagation the polymer chain grows, ${ }^{11}$

$$
M_{n}^{\bullet}+M \stackrel{k_{p}}{\longrightarrow} M_{n+1}^{\bullet} \text {, }
$$

where $k_{\mathrm{p}}$ is the rate constant of propagation and $M_{n}^{\bullet}$ and ${M_{n+1}}^{\bullet}$ are the growing macro-radical chains of $n$ and $(n+1)$ repeat monomeric units $(n \geq 1)$.

\section{Termination}

Termination can occur in three ways. Two of these, disproportionation and combination, involves two growing macroradicals interacting, i.e., the bimolecular termination mechanism.

$$
\begin{gathered}
M_{n}^{\bullet}+M_{m}^{\cdot} \stackrel{k_{t c}}{\longrightarrow} M_{n+m}, \\
M_{n}^{\bullet}+M_{m}^{\bullet} \stackrel{k_{t d}}{\longrightarrow} M_{n}+M_{m},
\end{gathered}
$$

where $k_{\mathrm{tc}}$ and $k_{\mathrm{td}}$ are the rate constants of combination and disproportionation termination respectively. $M_{n}, M_{m}$ and $M_{n+m}$ represent terminated chains which have no radical tip, i.e., a dead polymer. In this analysis, $k_{\mathrm{tc}}$ and $k_{\mathrm{td}}$ will be treated as a single lumped rate constant, $k_{\mathrm{t}}=k_{\mathrm{tc}}+k_{\mathrm{td}}\left(\mathrm{cm}^{3} \mathrm{~mol}^{-1} \mathrm{~s}^{-1}\right)$, as the mode of termination does not effect the polymerization kinetics. ${ }^{13}$

A third possible termination mechanism involves primary radical termination. ${ }^{13,16}$

$$
M_{n}^{\bullet}+R^{\bullet} \stackrel{k_{\text {tp }}}{\longrightarrow} M_{n} R,
$$

where $k_{\mathrm{tp}}$ is the rate constant of primary radical termination. In this step, a growing macro-radical chain reacts with a primary radical (initiator radical) leading once again to the production of inactive or dead polymer chains. ${ }^{13}$

\section{Inhibition}

Inhibitors are chemicals which react with the initiating and propagating radical species by rapidly removing or scavenging these radicals. Polymerization is completely halted until they are all consumed. ${ }^{12}$ Several possible inhibitor reaction mechanisms are listed below: 


$$
\begin{gathered}
\text { Dye } e^{*}+Z \stackrel{k_{z, D}}{\longrightarrow} \text { LeucoDye }+Z^{*}, \\
R^{\bullet}+Z \stackrel{k_{z, R^{\bullet}}}{\longrightarrow}\left(R+Z^{\bullet} \text {, and/or } R Z^{\bullet}\right), \\
M_{n}^{\bullet}+Z \stackrel{k_{z, M^{\bullet}}}{\longrightarrow}\left(M_{n}+Z^{\bullet} \text {, and/or } M_{n} Z^{\bullet}\right),
\end{gathered}
$$

where $Z$ is the inhibitor species, e.g., oxygen, $D y e^{*}$ is the excited photosensitiser, $Z$ is the concentration of singlet oxygen, ${ }^{12,15-17}$ and $k_{z, D}, k_{z, M}$, and $k_{z, R^{*}}$ are the rate constants of inhibition of the photosensitiser, the macro-radicals and the primary radicals respectively. Inhibition leads to a dead band at the start exposure, i.e., no initial grating formation during exposure. The effects of inhibitors are especially significant when lower exposure intensities are used, for example when large areas must be exposed or short pulses must be used. ${ }^{15}$ In order to further simplify the reaction modelling, we assume that $Z^{*}, R Z^{\bullet}$ and $M_{n} Z^{\bullet}$ do not reinitiate polymerization and that they terminate without regeneration. ${ }^{13,15}$ In addition, $Z^{*}, R-Z^{*}$ and $M_{\mathrm{n}}-Z^{*}$ are assumed to play no further part in grating formation, i.e., no further chain transfer mechanisms involve them. Furthermore, in the sealed material layer, it is assumed that the inhibitors are rapidly consumed at the start of the exposure. ${ }^{18}$

\section{Chain Transfer Mechanism}

In many polymerization systems, the average polymer weight is observed to be lower than predicted by the chain transfer reaction. ${ }^{8,12,19-23}$ Generally, the chain transfer process causes the premature termination of a growing macroradical chain and arises because of the presence of CTA. ${ }^{12}$ Due to this reaction, a new radical is produced which is referred as a re-initiator. This re-initiator reacts with a monomer molecule to initiate a new growing macro-radical chain. The chain transfer reactions can be written as

$$
\begin{gathered}
M_{n}^{\bullet}+(R I-X) \stackrel{k_{r r, S}}{\longrightarrow}\left(M_{n}-X\right)+R I^{\bullet}, \\
R I^{\bullet}+M \stackrel{k_{r i}}{\longrightarrow}\left(R I-M^{\bullet}\right),
\end{gathered}
$$

where $R I-X$ is the chain transfer agent, $-X$ is the atom or species transferred and $R I^{*}$ is the re-initiator which has a radical tip. $k_{\mathrm{tr}, \mathrm{S}}$ and $k_{\mathrm{ri}}$ are the transfer rate constant to chain-transfer agent and the re-initiation rate constant respectively Due to the premature termination reaction with the chain transfer agent, $R I-X$, the propagating polymer chains will stop growing earlier than they would have if the CTA was not present. We assume that the free radical $R I-M^{*}$ produced can be treated as acting chemically identical to a chain initiator $M^{*}$. Therefore the re-initiator, $R I^{*}$, simply initiates a new growing chain with a radical tip $M$. Thus, while the polymer chains are shortened, the amount of monomer polymerized and the rate of polymerization can remain high.

\section{$2.2 \quad$ Absorption}

During holographic grating formation in the AA/PVA based photopolymer, photosensitisation and initiation depend on the type and concentrations of both the dye and electron donor. During appropriate illumination of such a layer, sensitised with a xanthene or thiazine type dye, ${ }^{11,18}$ the photosensitiser absorbs a photon and is promoted into an excited state. As described in Eq. (1), an initiating species $R^{*}$ is produced by the reaction between an excited dye molecule and an electron donor molecule [Triethanolamine (TEA), $\left.\mathrm{C}_{6} \mathrm{H}_{15} \mathrm{NO}_{3}\right] .{ }^{11}$ The major reactions taking place during this step are as follows: ${ }^{15}$

$$
\text { Dye }+h v \stackrel{k_{a}}{\longrightarrow} D y e^{*}
$$




$$
\begin{gathered}
D y e^{*}+Z \stackrel{k_{z, D}}{\longrightarrow} \text { Leuco Dye, } \\
\text { Dye } \stackrel{k_{r}}{\longrightarrow} \text { Dye, } \\
D y e^{*}+E D \stackrel{k_{d}}{\longrightarrow} R^{\bullet}+H^{+}+\text {Dye } \\
E D+\text { HDye } \stackrel{k_{b}}{\longrightarrow} H_{2} \text { Dye }+E D_{\mathrm{int}},
\end{gathered}
$$

where Dye represents the photosensitiser (dye) species, $E D$ is the co-initiator (electron donor) and $Z$ is the inhibitor. $H D y e^{\bullet}$ represents a radicalised dye, which has abstracted a hydrogen ion from the co-initiator, and $\mathrm{H}_{2} \mathrm{Dye}$ is the di-hydro transparent form of the dye, i.e., a bleached transparent form of the dye molecule. $E D_{\text {int }}$ is an intermediate form of the co-initiator. ${ }^{11} k_{\mathrm{a}}\left(\mathrm{s}^{-1}\right)$ is the rate of production of excited state photosensitiser; $k_{\mathrm{z}, D}\left(\mathrm{~cm}^{3} / \mathrm{mol} \mathrm{s}\right)$ is the inhibition rate constant associated with the reaction of the inhibitor with the excited dye molecules, i.e., the formation of Leuco Dye; $k_{\mathrm{r}}$ $\left(\mathrm{s}^{-1}\right)$ is the rate of recovery or regeneration of photosensitiser back to the ground (absorbing) state; $k_{\mathrm{d}}\left(\mathrm{cm}^{3} / \mathrm{mol} \mathrm{s}\right)$ is the rate of dissociation of the initiator, and $k_{\mathrm{b}}$ is the rate constant of bleaching. In Eq. (6d), the dye radical formed abstracts a hydrogen molecule from the TEA free radical.

Applying the Beer-Lambert law, the rate of production of the excited state photosensitiser in Eq (6a) can be expressed as $k_{a}=\phi \varepsilon d I_{0}^{\prime}\left(\mathrm{s}^{-1}\right),{ }^{15,18}$ where $\phi$ (mol/Einstein) is the quantum efficiency of the reaction, $\varepsilon\left(\mathrm{cm}^{2} / \mathrm{mol}\right)$ is the molar absorptivity co-efficient of the dye, $d$ is the material layer thickness and $I_{0}^{\prime}\left(\right.$ Einstein $/ \mathrm{cm}^{2} \mathrm{~s}$ ) is the incident intensity corrected for Fresnel and scattering losses using the factor $T_{\mathrm{sf}}$. The exposure intensity $I_{0}$ is typically measured in units of $\mathrm{mW} / \mathrm{cm}^{2}$, and must be converted into the units of Einstein $/ \mathrm{cm}^{2} \mathrm{~s}$. This conversion can be performed using, $I_{0}{ }^{\prime}=\frac{T_{s f} B I_{0}}{d}\left(\frac{\lambda}{N_{a} h c}\right)$, where $\lambda(\mathrm{nm})$ is the wavelength of incident light, $N_{\mathrm{a}}\left(\mathrm{mol}^{-1}\right)$ is Avogadro's constant, $c(\mathrm{~m} / \mathrm{s})$ is the speed of light, and $h$ (Js) is Plank's constant. $B=1-e^{-\varepsilon_{0} d}$, is the absorptive fraction which determines a material layer's initial absorptive capacity and is a function of the dye's initial concentration, $\left[A_{0}\right]\left(\mathrm{mol} / \mathrm{cm}^{3}\right){ }^{15}$ The evolution of the concentration of the excited state dye, Dye $e^{*}$, can be determined for a given exposure intensity and initial photosensitiser concentration assuming $\phi, \varepsilon, d$ and $T_{\mathrm{sf}}$ are known.

\subsection{NPDD Model}

We assume illumination by a spatial distribution of irradiance $I(x, t)=I_{0}^{\prime}[1+V \cos (K x)]$, where $V$ is the fringe visibility and $K=2 \pi / \Lambda$, where $\Lambda$ is the grating period., ${ }^{9,24}$ The chemical equations, presented in Eq. (6), describe in detail primary radical production, which was previously summarised in Section 2.1. We now derive a set of coupled differential equations representing the spatial and temporal evolutions of the material concentrations associated with Eq. (6). Combining these with those previously presented in references, ${ }^{15,18}$ a more complete set of governing rate equations, are now presented:

$$
\frac{d[\text { Dye }(x, t)]}{d t}=-k_{a}[\text { Dye }(x, t)]+k_{r}\left[\text { Dye } e^{*}(x, t)\right]
$$




$$
\frac{d\left[D y e^{*}(x, t)\right]}{d t}=k_{a}[\operatorname{Dye}(x, t)]-k_{r}\left[D y e^{*}(x, t)\right]-k_{d}\left[D y e^{*}(x, t)\right][E D(x, t)]
$$$$
-k_{z, D}\left[D y e^{*}(x, t)\right][Z(x, t)]
$$

$$
\frac{d[E D(x, t)]}{d t}=-k_{d}\left[D y e^{*}(x, t)\right][E D(x, t)]-k_{b}[\operatorname{HDye} \cdot(x, t)][E D(x, t)],
$$

$$
\begin{gathered}
\frac{d\left[H D y e^{\bullet}(x, t)\right]}{d t}=k_{d}\left[D y e^{*}(x, t)\right][E D(x, t)]-k_{b}\left[H D y e^{\bullet}(x, t)\right][E D(x, t)], \\
\frac{d[Z(x, t)]}{d t}=\frac{d}{d x}\left\{D_{Z}(x, t) \frac{d[Z(x, t)]}{d x}\right\}-k_{z, D}\left[D y e^{*}(x, t)\right][Z(x, t)]-k_{z, R^{\bullet}}[Z(x, t)]\left[R^{\bullet}(x, t)\right] \\
-k_{z, M} \cdot[Z(x, t)][M \cdot(x, t)]+\tau_{z}\left\{Z_{0}-[Z(x, t)]\right\} .
\end{gathered}
$$

As indicated in Eq. (11), the non-uniform recording irradiance gives rise to concentration gradients, which result in the diffusion of inhibitor from the dark regions into the bright illuminated regions. Eq. (11) is derived based on the assumption that the oxygen is relatively free to diffuse, and does so rapidly, resulting in a one-dimensional standard diffusion equation for the concentration of inhibitor. ${ }^{15,25}$ As in the previous analysis, ${ }^{12-15}$ in Eq. (11), it is assumed that the effect of inhibition during exposure is caused by the initially dissolved oxygen present within the photopolymer layer and replenishing oxygen from the surrounding environment. In such an unsealed material layer case, we introduced the parameter, $\tau_{\mathfrak{z}}$, to represent the rate of replenishing of oxygen into the material layer from the surround environment. Also, we assume that $k_{z}=k_{z, R^{*}}=k_{z, M}$ and $k_{z, D}<<k_{z}$, as the reactivity of oxygen with the excited state form of the photosensitiser will be much lower than with $R^{*}$ or $M^{*} .^{12}[Z(x, t)]$ is the instantaneous inhibitor concentration, $D_{Z}(x, t)$ is the diffusion rate of the inhibitor in the dry material layer, and $Z_{0}\left(\mathrm{~mol} / \mathrm{cm}^{3}\right)$ is the initial dissolved inhibitor concentration. In addition, the diffusion of dye molecules (due to the concentration gradient) from the dark regions into the bright regions will eventually take place over an appreciably long time. ${ }^{26}$ But clearly the dye concentration following such diffusion can not be larger than its initial value. We assume that the effects of dye diffusion are negligible in this paper.

The equation governing the concentration of primary radicals is given by

$$
\begin{array}{r}
\frac{d\left[R^{\bullet}(x, t)\right]}{d t}=k_{d}\left[D y e^{*}(x, t)\right][E D(x, t)]-k_{i}\left[R^{\bullet}(x, t)\right][u(x, t)]-k_{t p}\left[R^{\bullet}(x, t)\right]\left[M^{\bullet}(x, t)\right] \\
-k_{z}\left[R^{\bullet}(x, t)\right][Z(x, t)],
\end{array}
$$

where $u(x, t)$ is the free-monomer concentration. This equation states that the contributions to the rate of change of the primary radical concentration includes the generation of primary radicals by photon absorption, and the amounts removed by: (a) the initiation of macro-radicals, (b) primary termination with growing polymer chains, and (c) inhibition by oxygen.

The equation governing the macro-radical concentration is then 


$$
\begin{aligned}
& \frac{d\left[M^{\bullet}(x, t)\right]}{d t}=k_{i}\left[R^{\bullet}(x, t)\right][u(x, t)]-k_{t}\left[M^{\bullet}(x, t)\right]^{2}-k_{t p}\left[R^{\bullet}(x, t)\right]\left[M^{\bullet}(x, t)\right] \\
& -k_{z}[Z(x, t)]\left[M^{\bullet}(x, t)\right] \text {, }
\end{aligned}
$$

The generation term in this equation previously appears as the removal term, arising due to macro-radical initiation, in Eq. (12). The removal terms include the effects of both types of termination mechanism, i.e., primary and bimolecular (the squared term), and the effects of inhibition. ${ }^{12-15}$

The co-sinusoidal irradiance leads to monomer concentration gradients, that result in monomer diffusion from the dark regions into the monomer depleted exposed regions. The polymer concentration distribution produced by the exposure provides the permanent modulation of refractive index in the material, i.e., the holographic grating. We represent the monomer concentration using the following $1 \mathrm{D}$ equation, ${ }^{15}$

$$
\frac{d[u(x, t)]}{d t}=\frac{d}{d x}\left\{D_{m}(x, t) \frac{d[u(x, t)]}{d x}\right\}-k_{i}\left[R^{\bullet}(x, t)\right][u(x, t)]-\int_{-\infty}^{\infty} k_{p}\left[M^{\bullet}\left(x^{\prime}, t\right)\right]\left[u\left(x^{\prime}, t\right)\right] G\left(x, x^{\prime}\right) d x^{\prime},
$$

where $D_{\mathrm{m}}(x, t)$ represents the monomer diffusion rate, and $G\left(x, x^{\prime}\right)$ is the non-local material spatial response function given by:

$$
G\left(x, x^{\prime}\right)=\frac{1}{\sqrt{2 \pi \sigma}} \exp \left[\frac{-\left(x-x^{\prime}\right)^{2}}{2 \sigma}\right]
$$

in which $\sigma$ is the non-local response parameter. The non-local spatial response function represents the effect of initiation at a location $x$ ' on the amount of monomer polymerized at location $x^{\left[{ }^{[6-8,13-15,18,27,28]}\right.} \sqrt{\sigma}$ represents the nonlocal response length, which is related to the average polymer chain length. ${ }^{27}$

The equation governing the polymer concentration is

$$
\frac{d[N(x, t)]}{d t}=-\frac{d}{d x}\left\{D_{N}(x, t) \frac{d[N(x, t)]}{d x}\right\}+\int_{-\infty}^{\infty} k_{p}\left[M \cdot\left(x^{\prime}, t\right)\right]\left[u\left(x^{\prime}, t\right)\right] G\left(x, x^{\prime}\right) d x^{\prime},
$$

where $D_{\mathrm{N}}(x, t)$ represents the polymer diffusion rate. If the polymer chains are not cross-linked sufficiently, they will tend to diffuse out of the exposed regions where they are formed. ${ }^{8}$ This process will result in a decay of the grating strength with time. However, in this paper, we assume that there is sufficient cross-linking and therefore $D_{\mathrm{N}}(x, t)=0$, i.e., very stable gratings are formed.

\subsection{Model Development}

In order to begin to examine the effects of the presence of CTA on the material non-local response length, $\sqrt{\sigma}$, we introduce a rate equation governing the CTA concentration:

$$
\frac{d[C T A(x, t)]}{d t}=\frac{d}{d x}\left\{D_{C T A}(x, t) \frac{d[C T A(x, t)]}{d x}\right\}-k_{t r, S}[C T A(x, t)]\left[M^{\bullet}(x, t)\right] .
$$


It should be noted that, in the following analysis, which is based on the assumptions stated earlier, we only consider chain transfer to chain-transfer agent, i.e., the chain transfer constants for monomer and initiator are assumed negligible. To further simplify the analysis in this paper, we assume that $k_{\mathrm{tr}}=k_{\mathrm{tr}, \mathrm{CTA}}$ and that the CTA diffusion rate, $D_{\mathrm{CTA}}$, is similar to the diffusion rate of monomer, $D_{\mathrm{m}}$, as their molecular weights are similar in the cases examined, i.e., $D_{\mathrm{CTA}} \approx D_{\mathrm{m}}$.

The equation governing the re-initiator concentration is

$\frac{d\left[R I^{\bullet}(x, t)\right]}{d t}=k_{t r}[C T A(x, t)]\left[M^{\bullet}(x, t)\right]-k_{r i}\left[R I^{\bullet}(x, t)\right][u(x, t)]$,

where $R I^{*}$ denotes the re-initiator concentration. The generation term here is the removal term arising in Eq. (5) due to the chain transfer process. Since it is assumed that the initiator radical, $R^{\circ}$, dominates the primary termination and inhibition processes, we only consider how the re-initiator, $R I^{\circ}$, reacts with the monomer.

Furthermore the chain transfer and re-initiation reactions effect the variation of macro-radical, $\left[M^{*}\right]$, and monomer, $[u]$, concentrations. Therefore Eq. (13) and (14) must be generalised:

$$
\begin{aligned}
& \frac{d\left[M^{\bullet}(x, t)\right]}{d t}= k_{i}\left[R^{\bullet}(x, t)\right][u(x, t)]+k_{r i}\left[R I^{\bullet}(x, t)\right][u(x, t)]-k_{t}\left[M^{\bullet}(x, t)\right]^{2} \\
&-k_{t p}\left[R^{\bullet}(x, t)\right]\left[M^{\bullet}(x, t)\right]-k_{z}[Z(x, t)]\left[M^{\bullet}(x, t)\right]-k_{t r}[C T A(x, t)]\left[M^{\bullet}(x, t)\right], \\
& \frac{d[u(x, t)]}{d t}=\frac{d}{d x}\left\{D_{m}(x, t) \frac{d[u(x, t)]}{d x}\right\}-k_{i}\left[R^{\bullet}(x, t)\right][u(x, t)] \\
&-\int_{-\infty}^{\infty} k_{p}\left[M^{\bullet}\left(x^{\prime}, t\right)\right]\left[u\left(x^{\prime}, t\right)\right] G\left(x, x^{\prime}\right) d x^{\prime}-k_{r i}[R I \bullet(x, t)][u(x, t)] .
\end{aligned}
$$

All the concentrations appearing in Eqs (7-12) and (16-20), will be periodic even functions of $x$ and can therefore be written as Fourier series, i.e., $[X(x, t)]=\sum_{j=0}^{\infty} X_{j}(t) \cos (j K x)$, where $X$ represents the particular species, i.e., Dye, Dye , $E D, H D y e^{\bullet}, R^{\bullet}, M^{\bullet}, C T A, R I^{\bullet}, u, N$ and $Z$. A set of first-order coupled differential equations can then be obtained by gathering the coefficients of the various co-sinusoidal spatial components and writing the equations in terms of these time varying spatial harmonic amplitudes, $X_{\mathrm{j}}(t)$. Assuming that harmonics of order greater than $j=3$, are negligible. ${ }^{13-15}$ These coupled equations must be solved for the initial concentration values given in Section 5, i.e., when $t=0$,

$$
\begin{gathered}
{\left[\text { Dye }_{j \geq 0}^{*}\right]=\left[H D_{j \geq 0}^{\cdot}\right]=\left[R_{j \geq 0}^{\cdot}\right]=\left[M_{j \geq 0}^{\cdot}\right]=\left[R I_{j \geq 0}^{\cdot}\right]=\left[N_{j \geq 0}\right]=0, \text { and }} \\
{\left[\text { Dye }_{j>0}\right]=\left[Z_{j>0}\right]=\left[u_{j>0}\right]=\left[E D_{j>0}\right]=\left[C T A_{j>0}\right]=0 .}
\end{gathered}
$$

In the radical chain polymerization system, ${ }^{8,12}$ the polymerization rate can be expressed as:

$$
R_{P}=k_{p}[M \cdot(x, t)][u(x, t)] .
$$


The polymerization rate, $R_{\mathrm{p}}$, is also related to the number-average degree of polymerization, $D P_{\mathrm{n}}$. $D P_{\mathrm{n}}$ is defined as the average number of structural units per polymer chain. It indicates the average length and therefore molecular weight of the polymer chains formed. According to the Mayo Equation: ${ }^{12,19}$

$$
\frac{1}{D P_{n}}=\frac{k_{t} R_{p}}{k_{p}^{2}[u]^{2}}+C_{u}+C_{C T A} \frac{[C T A]}{[u]}+C_{I} \frac{[I]}{[u]}
$$

This quantifies the effect of the various chain transfer reactions on the number-average degree of polymerization. [u], $[C T A],[I]$, represent the concentrations of monomer, chain transfer agent and initiator, respectively. The chain-transfer constants, $C_{\mathrm{u}}, C_{\mathrm{CTA}}$ and $C_{\mathrm{I}}$, for each particular substance are defined as the ratios of the rate constants for chain transfer of a propagating radical with that substance to the propagation rate constant, $k_{\mathrm{p}}$. They are given by:

$$
C_{u}=\frac{k_{t r, u}}{k_{p}}, C_{C T A}=\frac{k_{t r, C T A}}{k_{p}} \text {, and } C_{I}=\frac{k_{t r, I}}{k_{p}},
$$

where $k_{\mathrm{tr}, \mathrm{u}}, k_{\mathrm{tr}, \mathrm{CTA}}, k_{\mathrm{tr}, \mathrm{I}}$ represent rate constants for chain transfer to monomer, chain transfer agent and initiator respectively. For the case examined here the chain transfer constants to monomer and initiator, can be omitted as they are typically very low for acrylamide, ${ }^{29,30}$ and therefore Eq. (23) can be simplified to:

$$
\frac{1}{D P_{n}}=\frac{k_{t} R_{p}}{k_{p}^{2}[u]^{2}}+C_{C T A} \frac{[C T A]}{[u]},
$$

which will be discussed in detail in next section.

\section{NUMERICAL RESULTS}

The predictions of the model presented in this paper are now discussed. All kinetic parameter values are assigned appropriate values, which are typical for the AA/PVA photopolymer material examined. The simulations are performed retaining four spatial concentration harmonics and therefore four coupled equations are solved for the initial conditions given in Eq. (29). In all cases, $[U]_{0}=2.83 \times 10^{-3} \mathrm{~mol} / \mathrm{cm}^{3},[A]_{0}=1.22 \times 10^{-6} \mathrm{~mol} / \mathrm{cm}^{3},[E D]_{0}=3.18 \times 10^{-3} \mathrm{~mol} / \mathrm{cm}^{3},[C T A]_{0}$ $=1 \times 10^{-6} \mathrm{~mol} / \mathrm{cm}^{3}$, and $[Z]_{0}=1 \times 10^{-8} \mathrm{~mol} / \mathrm{cm}^{3}, 8,13,14$ where $[U]_{0},[A]_{0},[E D]_{0},[C T A]_{0}$ and $[Z]_{0}$ represent the concentrations at $t=0$, of monomer, photosensitizer, electron donor, transfer agent and inhibitor, respectively. In this paper, we assume that time varying viscosity effects are negligible, i.e., $D_{\mathrm{m}, \mathrm{j}>0}=0$, and that, $D_{\mathrm{m} 0}=1.0 \times 10^{-10} \mathrm{~cm}^{2} / \mathrm{s}^{31,32}$ For an exposure intensity of $I_{0}=1 \mathrm{~mW} / \mathrm{cm}^{2}, \lambda=532 \mathrm{~nm}$ and thickness $d=100 \mu \mathrm{m}$, the absorption parameters are, $\varepsilon=1.43 \times 10^{8} \mathrm{~cm} / \mathrm{mol}^{2}$, $\phi=0.01 \mathrm{~mol} /$ Einstein and $T_{\mathrm{sf}}=0.76$. The oxygen diffusion coefficient is $D_{\mathrm{z}}(x, t)=D_{\mathrm{z}}=1.0 \times 10^{-8} \mathrm{~cm}^{2} / \mathrm{s}$ and $\tau_{\mathrm{z}}=0$, i.e., sealed layers are used. ${ }^{15}$ The typical rate constants used were: $k_{\mathrm{p}}=k_{\mathrm{i}}=1 \times 10^{7} \mathrm{~cm}^{3} / \mathrm{mol} \mathrm{s}, k_{\mathrm{t}}=3 \times 10^{8} \mathrm{~cm}^{3} / \mathrm{mol} \mathrm{s}, k_{\mathrm{tp}}=k_{\mathrm{t}} \times$ $10, k_{\mathrm{d}}=k_{\mathrm{b}}=1.6 \times 10^{3} \mathrm{~cm}^{3} / \mathrm{mol} \mathrm{s}, k_{\mathrm{tr}}=1 \times 10^{7} \mathrm{~cm}^{3} / \mathrm{mol} \mathrm{s}, k_{\mathrm{ri}}=1 \times 10^{6} \mathrm{~cm}^{3} / \mathrm{mol} \mathrm{s}, k_{\mathrm{z}}=3 \times 10^{12} \mathrm{~cm}^{3} / \mathrm{mol} \mathrm{s}$, and $k_{\mathrm{r}}=1.2 \times 10^{-3} \mathrm{~s}$ $1.12,15,18,33$ Assuming typical recording conditions for an unslanted transmission type volume holographic grating, i.e., period $\Lambda=400 \mathrm{~nm}$ and fringe visibility $V=1$, the resulting predictions of the temporal and spatial behaviour of the photochemical processes are now examined. 


\section{$R_{\mathrm{p}}\left(\mathrm{mol} / \mathrm{cm}^{3} \mathrm{~s}\right)$ \\ [ $\left.\times 10^{-4}\right]$}

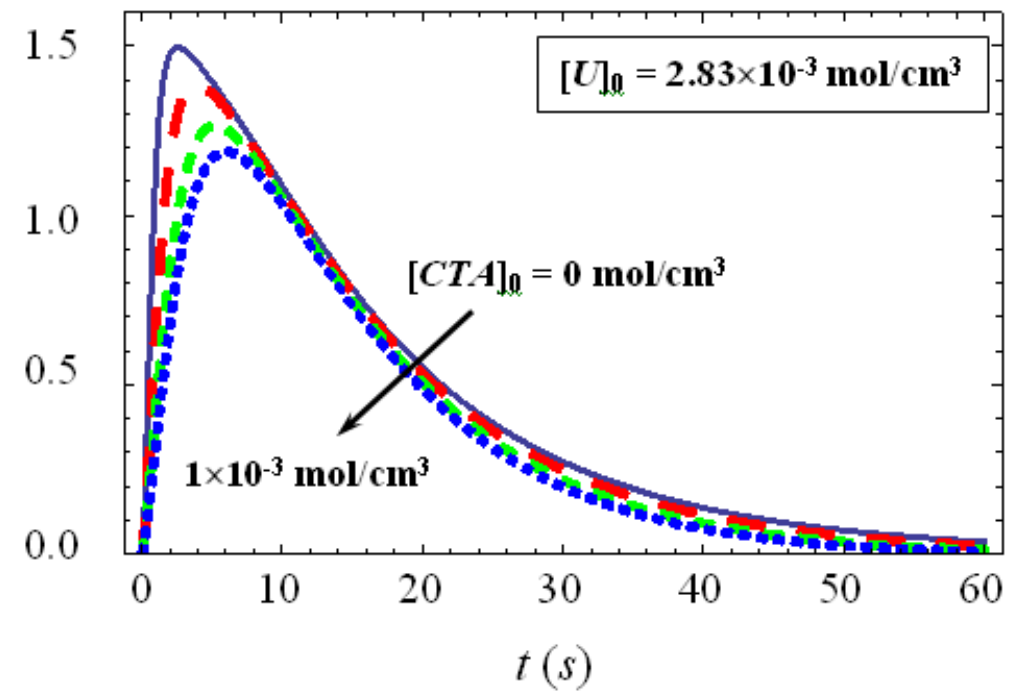

Figure 1. Simulation of the variation of polymerization rate, $R_{\mathrm{p}}\left[\mathrm{mol} / \mathrm{cm}^{3} \mathrm{~s}^{1}\right]$, during exposure for four different initial concentration of CTA, $[C T A]_{0}\left[\mathrm{~mol} / \mathrm{cm}^{3}\right]$ : $\left\{0\right.$ (solid), $3 \times 10^{-4}$ (long dashed); $7 \times 10^{-4}$ (dashed) and $1 \times 10^{-3}$ (short dashed) $\}$. Figure 1 shows the polymerization rate, $R_{\mathrm{p}}$, which appears in Eq. (22), simulated for four different initial concentrations of CTA, $[C T A]_{0}=\left\{0,3 \times 10^{-4}, 7 \times 10^{-4}\right.$, and $\left.1 \times 10^{-3}\right\} \mathrm{mol} / \mathrm{cm}^{3}$. As the initial concentration of CTA is reduced to zero, it can be seen that the polymerization rate increases more rapidly towards a higher maximum value and then decreases at a faster rate. From Figure 1, it should be noted that, for $[C T A]_{0}<[u]_{0}$, the effects of CTA on the polymerization rate are not very large, i.e., a similar number of monomer molecules will be consumed per unit time during grating formation.

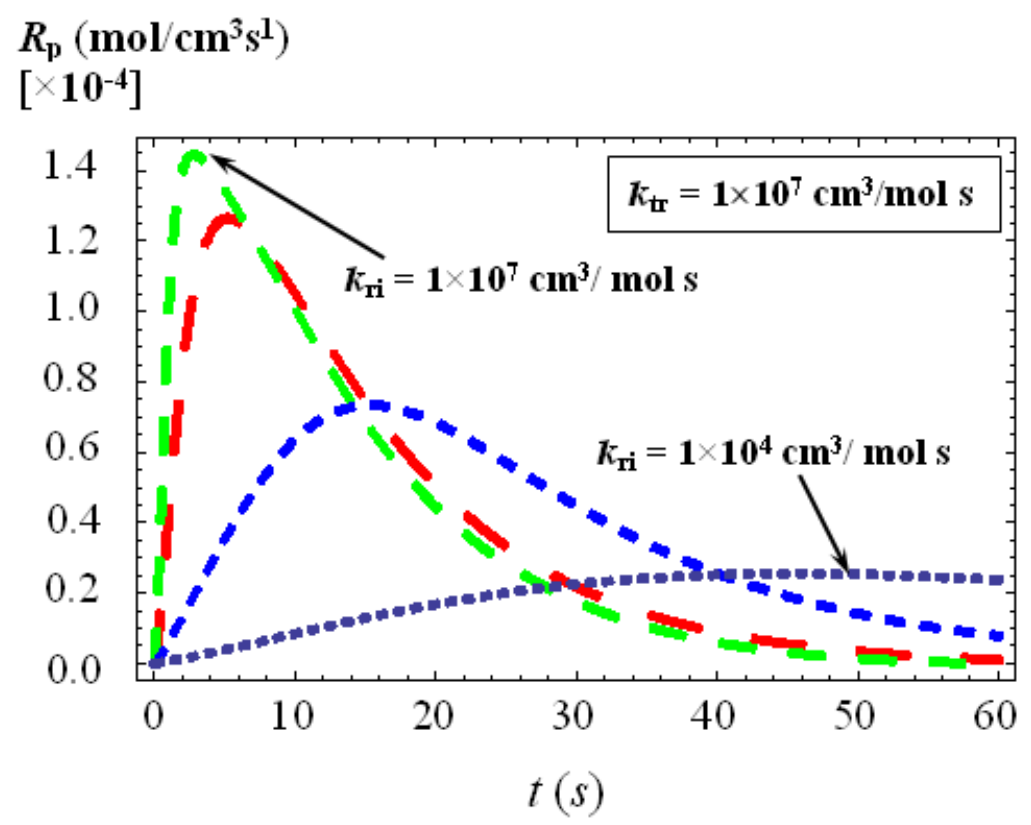

Figure 2. Simulation of variation of polymerization rate, $R_{\mathrm{p}}\left[\mathrm{s}^{-1}\right]$, during exposure for three different re-initiation rate, $k_{\mathrm{ri}}$ [ $\mathrm{cm}^{3} / \mathrm{mol} \mathrm{s}$ ]: $\left\{1 \times 10^{4}\right.$ (shorter dashed); $1 \times 10^{5}$ (short dashed); $1 \times 10^{6}$ (dashed) and $1 \times 10^{7}$ (long dashed) $\}$.

The effects of various CTA re-initiation rates, $k_{\mathrm{ri}}$, on the polymerization rate are shown in Figure 2, where the rate constant of chain transfer is $k_{\mathrm{tr}}=1 \times 10^{7} \mathrm{~cm}^{3} / \mathrm{mol} \mathrm{s}$ and four different re-initiation rates, $k_{\mathrm{ri}} \sim\left\{1 \times 10^{4}, 1 \times 10^{5}, 1 \times 10^{6}\right.$ and 
$1 \times 10^{7} \mathrm{~cm}^{3} / \mathrm{mol} \mathrm{s}$, are examined. Figure 2 clearly shows that the effect of a CTA on the polymerization rate is highly dependent on whether the re-initiation rate is comparable to that of the propagation rate, $k_{\mathrm{p}}=1 \times 10^{7} .{ }^{12}$ It can be seen that there is a large decrease in the polymerization rate, $R_{\mathrm{p}}$, as the re-initiation rate decreases. When the re-initiation rate, $k_{\mathrm{ri}}$, is comparable to the propagation rate, $k_{\mathrm{p}}$, one observes very little change in the polymerization rate. When the reinitiation rate, $k_{\mathrm{ri}}$, is slow compared to propagation rate, i.e., when $k_{\mathrm{ri}}<<k_{\mathrm{p}}$, one observes a large decrease in the polymerization rate, $R_{\mathrm{p}}$.

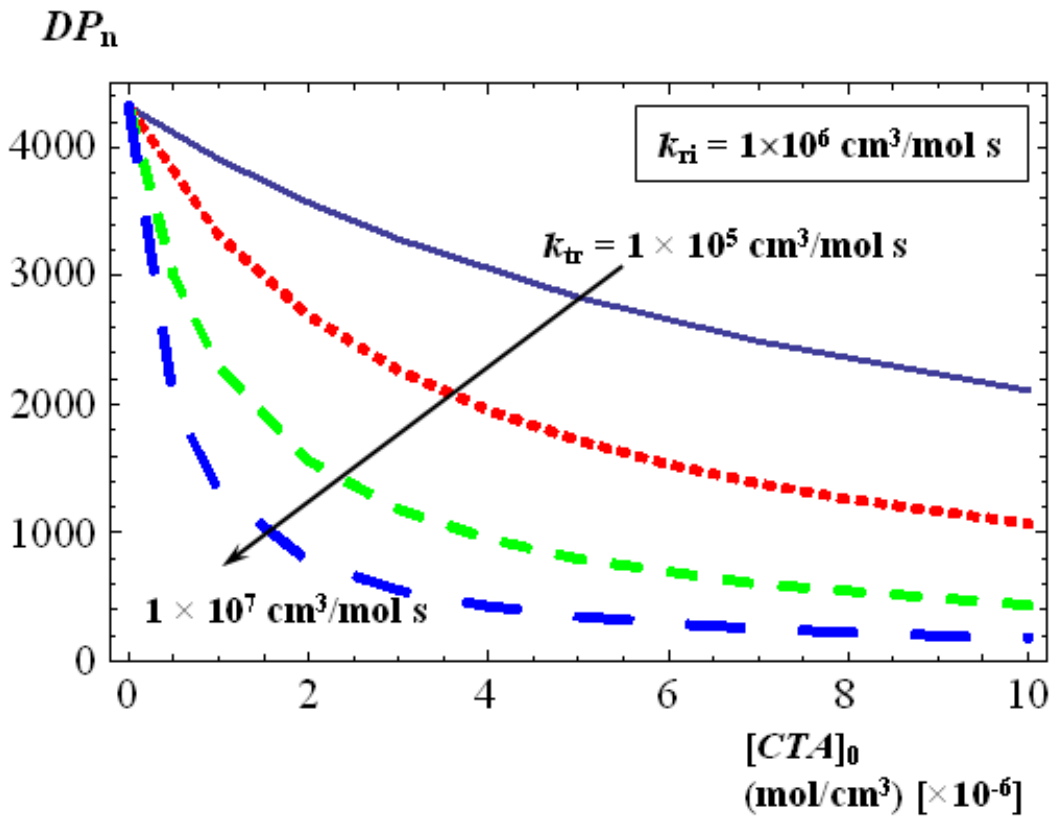

Figure 3. Effects of initial CTA concentration on number-average degree of polymerization, $D P_{\mathrm{n}}$, and contributions of various rate constants of chain transfer, $k_{\mathrm{tr}}$, [cm $\left./ \mathrm{mol} \mathrm{s}\right]:\left\{1 \times 10^{5}\right.$ (solid); $3 \times 10^{5}$ (short dashed); $1 \times 10^{6}$ (dashed) and $1 \times 10^{7}$ (long dashed)\}.

Different types of transfer agents will exhibit different kinetic behaviours, which result in variations in the polymerization rate and therefore changes to the number-average degree of polymerization, $D P_{n}{ }^{12}$ The results in Figure 3 demonstrate the effects of varying the initial CTA concentration on the $D P_{\mathrm{n}}$ when the re-initiation rate $k_{\mathrm{ri}}=1 \times 10^{6}$ $\mathrm{cm}^{3} / \mathrm{mol} \mathrm{s}$. For a particular type of CTA, i.e., when $k_{\mathrm{tr}}=1 \times 10^{7} \mathrm{~cm}^{3} / \mathrm{mol} \mathrm{s}$ (long dashed in Figure 3), it can be seen that increasing the initial CTA concentration leads to a rapid decrease in $D P_{\mathrm{n}}$ and that the $D P_{\mathrm{n}}$ value decreases more slowly to lowest value shown. This result indicates that, for an appropriate concentration of CTA, i.e., $1 \times 10^{-6}<[C T A]_{0}<4 \times 10^{-}$ ${ }^{6} \mathrm{~mol} / \mathrm{cm}^{3}, D P_{\mathrm{n}}$ is always reduced with the inclusion of CTA and that the reduction is larger for higher CTA concentrations. Furthermore, when $k_{\mathrm{tr}} \geq 1 \times 10^{7} \mathrm{~cm}^{3} / \mathrm{mol} \mathrm{s}$, the model predicts that at above some specific CTA concentration a threshold exists and further increases do not result in any further significant reduction in $D P_{n}$, i.e., when $[C T A]_{0}>4 \times 10^{-6} \mathrm{~mol} / \mathrm{cm}^{3}$. Ideally, one wishes to identify the least amount of CTA required in order to achieve the largest reduction in the number-average of polymerization, $D P_{n}$. We also note that, for the same initial CTA concentration, a reduction in $D P_{\mathrm{n}}$ also takes place for an increase in chain transfer kinetic value, $k_{\mathrm{tr}}$. Thus the addition of different types and concentrations of chain transfer agents are predicted to have different effects on the value of $D P_{\mathrm{n}}$ and therefore on the average polymer chain length in the otherwise identical photopolymer system. As discussed in Section 2 , the non-local response length, $\sqrt{\sigma}$, and the number-average degree of polymerization, $D P_{\mathrm{n}}$, are both related to the average polymer chain length. We would expect that any significant reduction in the number-average degree of polymerization, $D P_{\mathrm{n}}$, should be accompanied by a reduction in the non-local response length, $\sqrt{\sigma}$, and therefore by an improvement in the refractive index modulation, $n_{1}$, which can be recorded at high spatial frequencies in the material. 


\section{$n_{1}\left[\times 10^{-3}\right]$}

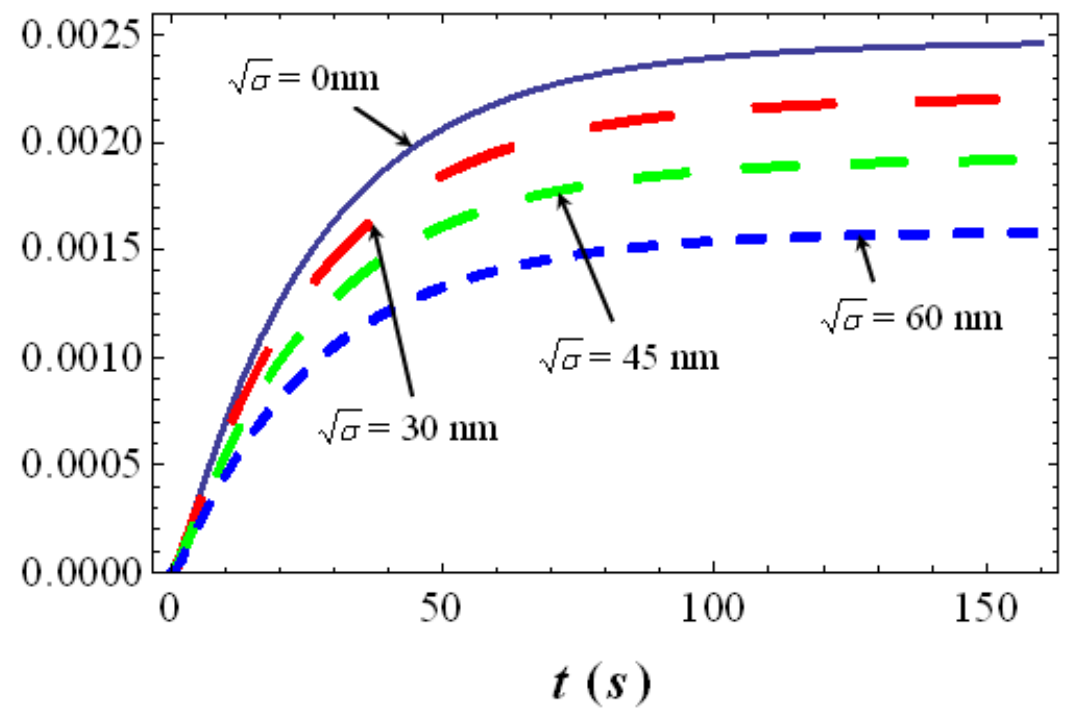

Figure 4. Simulations of the growth curves of refractive index modulation, $n_{1}$, at $\Lambda=400 \mathrm{~nm}$ for various values of nonlocal response length, $\sqrt{\sigma}$, [nm]: $\{0$ (solid); 30 (long dashed); 45 (dashed) and 60 (short dashed) .

In order to demonstrate the relationship between $\sqrt{\sigma}$ and $n_{1}$, Figure 4 shows four simulated growth curves of refractive index modulation, $n_{1}$, for four different values of $\sqrt{\sigma}$. In all cases the same typical rate constant values employed earlier in this section are used. We see that larger values of $\sqrt{\sigma}$, lead to lower saturation (maximum) values of $n_{1}$ for the same grating period. In another word, a lower $\sqrt{\sigma}$ value indicates more localized polymerization, involving the generation of shorter polymer chains, takes place during holographic grating formation.

$$
n_{1}^{\text {sat. }}\left[\times 10^{-3}\right]
$$

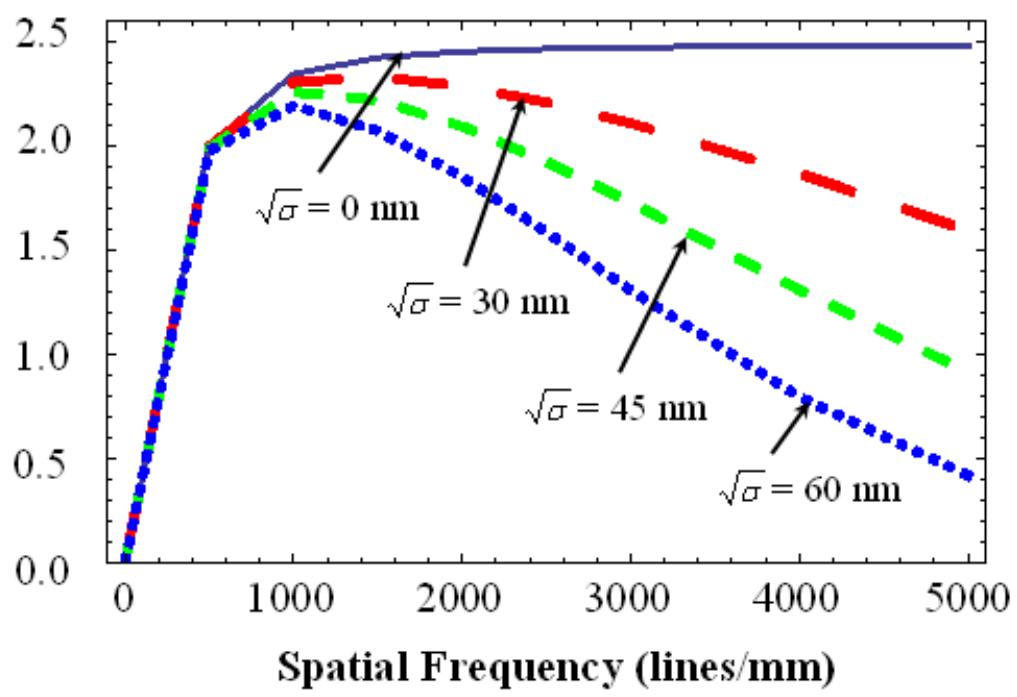

Figure 5. Simulations of the spatial frequency response of saturation refractive index modulation, $n_{1}$, variation, for various values of non-local response length, $\sqrt{\sigma}$, [nm]: $\{0$ (solid); 30 (long dashed); 45 (dashed) and 60 (short dashed) $\}$.

Figure 5 shows the saturation refractive index modulation, $n_{1}^{\text {sat }}$, plotted as a function of the grating spatial frequency, for the same grating parameter values, used for Figure 4. These results predict that lower $\sqrt{\sigma}$ values lead to a significant 
improvement in the high spatial frequency response of the material and therefore a reduction in the high spatial frequency roll-off observed experimentally. This is an important prediction of the NPDD model and motivates the study of the feasibility of applying chain transfer agents in free radical based photopolymer materials.

\section{CONCLUSIONS}

In this paper, we explored some of the photochemical and photo-physical predictions of the Non-local Photopolymerisation Driven Diffusion model with the aim of illustrating some trends of practical importance when attempting to optimise the performance of a photopolymer material. The implications of the predictions presented in this paper suggest that, utilising a chain transfer agent which has a relatively large chain transfer rate will leads to a more rapid decrease in $D P_{\mathrm{n}}$ and therefore a reduction in the non-local response length. It also should be noted that, for a particular type of CTA, there is a specific range of CTA concentration, which leads to a significant reduction in $D P_{\mathrm{n}}$. The prediction implies, when the addition of CTA is used for many applications, an appropriate CTA concentration must be taken into account.

\section{ACKNOWLEDGMENTS}

We acknowledge the support of the Irish Research Council for Science, Engineering and Technology through the Empower Postdoctoral research scholarship. We also acknowledge the support of Enterprise Ireland and Science Foundation Ireland through the national development plan.

\section{REFERENCES}

[1] K. Curtis, L. Dhar, L. Murphy, and A.Hill, Future Developments, in Holographic Data Storage: From Theory to Practical Systems (Wiley, 2010).

[2] C. Ye and R. R. McLeod, “GRIN lens and lens array fabrication with diffusion-driven photopolymer," Opt. Lett. 33(22), 2575-2577, (2008).

[3] A. Sato, M. Scepanovic, and R. Kostuk, "Holographic edge-illuminated polymer Bragg gratings for dense wavelength division optical filters at 1550 nm,” Appl. Opt. 42(5), 778-784, (2003).

[4] O. Kashin, E. Tolstik, V. Matusevich, and R. Kowarschik, "Numerical investigation of the (1+1)D self-trapping of laser beams in polymeric films based on polymethylmethacrylate and phenanthrenequinone,” J. Opt. Soc. Am. B 26(11), 2152-2156, (2009).

[5] F. Bruder and T. Facke, Int. “Materials in optical data storage,” J. Mat. Res. 101(2), 199-215, (2010).

[6] J. T. Sheridan and J. R. Lawrence, "Nonlocal response diffusion model of holographic recording in photopolymer,” J. Opt. Soc. Am. A 17(6), 1108-1114, (2000).

[7] J. R. Lawrence, F. T. O’Neill, and J. T. Sheridan, "Adjusted intensity nonlocal diffusion model of photopolymer grating formation," J. Opt. Soc. Am. B 19(4), 621-629, (2002).

[8] M. R. Gleeson, D. Sabol, S. Liu, C. E. Close, J. V. Kelly, and J. T. Sheridan, "Improvement of the spatial frequency response of photopolymer materials by modifying polymer chain length,” J. Opt. Soc. Am. B 25(3), 396-406, (2008).

[9] G. H. Zhao and P. Mouroulis, “Diffusion-model of hologram formation in dry photopolymer materials,” J. Mod. Opt. 41(10), 1929-1939, (1994).

[10] J. T. Sheridan, M. R. Gleeson, C. E. Close, J. V. Kelly, "Optical response of photopolymer materials for holographic data storage applications,” J. Nanosci. Nanotechnol. 7(1), 232-242, (2007).

[11] J. R. Lawrence, F. T. O'Neill, and J. T. Sheridan, "Photopolymer holographic recording material," Optik 112(10), 449-463, (2001).

[12] G. Odian, Principles of Polymerization $4^{\text {th }}$ Edition, (Wiley, New York, 1991).

[13] M. R. Gleeson and J. T. Sheridan, "Non-local photo-polymerization kinetics including multiple termination mechanisms and dark reactions: Part I. Modelling,” J. Opt. Soc. Am. B 26(9), 1736-1745, (2009).

[14] M. R. Gleeson, S. Liu, R. R. McLeod, and J. T. Sheridan, "Non-local photo-polymerization kinetics including multiple termination mechanisms and dark reactions: Part II. Experimental Validation,” J. Opt. Soc. Am. B 26(9), 1746-1754, (2009).

[15] M. R. Gleeson, S. Liu, J. Guo and J. T. Sheridan, "Non-Local photo-polymerization kinetics including multiple termination mechanisms and dark reactions: Part III. Primary Radical Generation and Inhibition,” J. Opt. Soc. Am. B 27, 1804-1812 (2010). 
[16] S. Liu, M. R. Gleeson, J. Guo and J. T. Sheridan, “Optical characterization of photopolymers materials: Theoretical and experimental examination of primary radical generation,” Appl. Phys. B 100, 559-569 (2010).

[17] A. Fimia, N. Lopez, F. Mateos, R. Sastre, J. Pineda, and F. Amat-Guerri, "Elimination of oxygen inhibition in photopolymer systems used as holographic recording materials,” J. Mod. Opt. 40, No. 4, 699-706, (1993).

[18] S. Liu, M. R. Gleeson, J. Guo, and J. T. Sheridan, "High intensity response of photopolymer materials for holographic grating formation,” Macromolecules 43, 9462-9472, (2010).

[19] F. R. Mayo, "Chain Transfer in the Polymerization of Styrene: The Reaction of Solvents with Free Radicals,” J. Am. Chem. Soc. 65 (12), 2324-2329, (1943).

[20] L. A. Goretta and R. R. Otremba, U.S. Patent No. 4,307,215, (1981).

[21] H. A. Gartner, U.S. Patent No. 5,171,783, (1992).

[22] M. Fevola, R. Hester, and C. McCormack, "Molecular weight control of Polyacyrlamide with Sodium Formate as a Chain-Transfer Agent: Characterization via size Exclusion Chromatography/multi-angle laser light ccattering and determination of chain-Transfer constant,” J. Polym. Sci., Part A: Poly. Chem. 41, 560-568, (2003).

[23] F. Karasu, N. Arsu, and Y. Yagci, "2-Mercapto Thioxanthone as a Chain Transfer Agent in Free-Radical Polymerization” J. Appl. Poly. Sci. 103, 3766-3770, (2007).

[24] H. Kogelnik, “Coupled wave theory for thick holographic gratings,” Bell Sys. Tech. J. 48, 2909-2947, (1969).

[25] A. K. O’Brien and C. N. Bowman, "Modelling the effect of oxygen on photopolymerization kinetics," Macromol. Theory Simul. 15, 176-182, (2006).

[26] J. Guo, S. Liu, M. R. Gleeson, and J. T. Sheridan, "Study of photosensitizer diffusion in a photopolymer material for holographic applications,” J. Opt. Eng. 50(1), 015801, (2011).

[27] M. Doi, Introduction to Polymer Physics (Oxford, 1997), pp. 1-8.

[28] F. T. O’Neill, J. R. Lawrence, and J. T. Sheridan, "Comparison of holographic photopolymer materials by use of analytic nonlocal diffusion models,” Appl. Opt. 41, 845-852 (2002).

[29] A. Valdebenito and M. V. Encinas, "Thiophenols as chain transfer agents in the polymerization of vinyl monomers,” Polymer, 46, 10658-10662, (2005).

[30] T. Okaya, K. Kikuchi, and Y. Morii, "Polymerization of acrylamide in aqueous medium initiated with a redox system composed of cysteine and potassium bromate” Macromol Chem Phys, 198, 2027-2034, (1997).

[31] C. Neipp, S. Gallego, M. Ortuno, A. Marquez, M. L. Alvarez, A. Belendez, and I. Pascual, "First-harmonic diffusion-based model applied to a polyvinyl-alcohol-acrylamide-based photopolymer,” J. Opt. Soc. Am. B 20, 2052-2060 (2003)

[32] J. Lougnot, P. Jost, and L. Lavielle, "Polymers for holographic recording: VI. some basic ideas for modelling the kinetics of the recording process,” Pure and Appl. Opt. 6, 225-245 (1997).

[33] J. Brandrup, E. H. Immergut, and E. A. Grulke, Polymer Handbook $4^{\text {th }}$ Edition (Wiley, 1999). 